\title{
Small-Angle Neutron Scattering from Viscoelastic Polymer-Clay Solutions
}

\author{
Gudrun Schmidt, ${ }^{,+,, \S}$ Alan I. Nakatani, ${ }^{\dagger, \perp}$ Paul D. Butler, ${ }^{\ddagger}$ and Charles C. Han ${ }^{\dagger}$
}

National Institute of Standards and Technology, Gaithersburg, Maryland 20899, and Oak Ridge National Laboratory, Oak Ridge, Tennessee 37831

Received August 22, 2001; Revised Manuscript Received March 27, 2002

\begin{abstract}
The influence of shear on viscoelastic polymer-clay solutions was investigated by means of small-angle neutron scattering (SANS) under shear. SANS was used to measure the shear-induced orientation of polymer and platelets. With increasing shear rate an anisotropic scattering pattern devel oped. At higher shear rates, the scattering anisotropy increased due to the enhanced orientation of the clay platelets in the shear field. The clay platelets aligned by the flow in an unusual direction, with the surface normal parallel to the vorticity direction. SANS on regular samples (contrast between $\mathrm{D}_{2} \mathrm{O}$ and solution components) measured the shear-induced orientation of polymer and platelets. However, with contrast matching the orientation of the polymer alone could be detected. With increasing shear rate, clay particles oriented first (SANS on regular samples) and then polymer chains started to stretch (SANS on contrast matched samples). Cessation of shear led to fast recovery, demonstrating the system to be highly elastic.
\end{abstract}

\section{Introduction}

In recent years, organic-inorganic nanocomposite materials, which frequently exhibit synergistic behavior and hybrid properties derived from several components, have attracted the interest of a number of researchers. Nanocomposite polymeric materials in bulk ${ }^{1-6}$ and in solutions ${ }^{7-10}$ offer unique mechanical, el ectrical, optical , and thermal properties. Such property enhancements are induced not only by the physical presence of the filler but also by the interaction of the polymer with the filler and the state of dispersion. Most reinforcing agents such as fibers and fillers are large and scatter light, thus reducing light transmittance. Efficient particle dispersion combined with good interfacial adhesion is achieved in aqueous polymer-clay solutions. This property is maintained in the bulk material, which is the main reason for transparency. From a practical viewpoint, this allows the exciting possibility of developing strong yet transparent films, coatings, and membranes from a polymer-clay solution. Additionally, the large aspect ratio of clay platelets may lead to a supramolecular organization similar to other mesoscopic systems such as liquid crystalline polymers, surfactants, or block copolymers. Shear-induced structural changes in fluids containing anisotropic species are a very general problem encountered in polymer solutions, 11,12 liquid crystalline materials, ${ }^{11,13-15}$ block copolymer melts, ${ }^{16-19}$ and platelike clay solutions. ${ }^{10,20-24} \mathrm{~A}$ large body of literature exists on the flow behavior of clay solutions as well as polymer solutions. However, little is known about the influence of shear on viscoelastic clay-polymer solutions. ${ }^{25,26}$

Previous investigations on pure clay solutions proposed many structural models to explain the mesoscopic properties and shear behavior of aqueous clay solutions. Gelation effects have been described by van OIphen by

\footnotetext{
† National Institute of Standards and Technology.

₹ Oak Ridge National Laboratory.

§ Present address: Louisiana State University.

$\perp$ Present address: Rohm and Haas Company.

* To whom correspondence should be sent: e-mail gudrun@ Isu.edu.
}

an electrostatic attraction between the positively charged edges and negatively charged faces of the platelets, which resulted in a linked structure similar to a house of cards. 27,28 Monte Carlo simulations suggested that such a house of cards is indeed possible.29,30 Direct inspection of the gel phase by cryofracture, TEM, and SAXS showed correlated but well-separated particle populations. TEM indicated that clay particles are isolated and so far apart that they hardly ever touch.21,31 An alternative structure suggested by Norrish et al. explained the gel formation by a long-range electrostatic repulsion between interacting double layers. ${ }^{32}$

Rheological studies by Rand and Melton on gels formed by Kaolinite dispersions were interpreted in terms of edge-face, edge-edge, and face-face aggregated structures. ${ }^{9,33}$ I contrast, for montmorillonite solutions Rand et al. found no evidence for edge-face interactions from rheological measurements. ${ }^{34}$ L aponite is similar to montmorillonite, a threelayer hectorite type clay with magnesium ions in between the two silicate layers. However, the smaller diameter of Laponite results in a smaller aspect ratio (30:1) than for montmorillonite (100:1). Under appropriate conditions Laponite and montmorillonite platel ets may completely exfoliate in aqueous solutions. Saunders et al.,35 Gabriel et al.,36 Mourchild et al.,37 and Ramsay et al. ${ }^{10}$ found evidence for aligned, ordered structures in Laponite gels from birefringence and X-ray studies. These studies were done on quiescent samples. The textures of these birefringent clay samples as observed with polarizing microscopy are typical of a liquid crystalline nematic phase. These results were interpreted in terms of re pulsive electrostatic interactions. According to Mourchild et al., Laponite particles at the sol-gel transition interact and are not totally free to move separately. 21,31 One possible way to mi nimize the total Gibbs energy is to align neighboring particles in order to save free volume and reduce their excluded-volume interactions.

One more structure proposed for Laponite suspensions is the fractal network. Several groups have interpreted their light- and X-ray scattering experiments in terms of fractal dimension. Very often rheo- 
Table 1. Composition of Samples ${ }^{a}$

\begin{tabular}{lcc}
\hline sample & PEO \% mass fraction & LRD \% mass fraction \\
\hline PEO & 3 & 0 \\
LRD & 0 & 3 \\
LRD1 & 1 & 3 \\
LRD2 & 2 & 3 \\
LRD3 & 3 & 3 \\
LRD4 & 4 & 3 \\
LRD5 & 5 & 3
\end{tabular}

a Relative mass error: $<1 \%$.

logical properties of Laponite suspensions have been related to the fractal structure measured by light scattering. ${ }^{37-39}$ Recent studies by Bonn et al. ${ }^{40}$ have concluded that the fractality of pure Laponite solutions is dependent on the specific way the suspension was prepared. According to these authors, the fractal structure is an artifact, which is most probably due to an incomplete dissolution of clustered particles. A complete dispersion of Laponite particles in solution showed characteristics of a colloidal glass. ${ }^{40}$

Bihannic and co-workers described a direct visualization of montmorillonite gels by X-ray microscopy. ${ }^{41}$ They observed an unexpected superstucture and long-range organization over distances at least 2 orders of magnitude larger than the dimensions of individual montmorillonite platelets.

SANS scattering studies on pure Laponite as well as montmorillonite sol utions under static and shear conditions have been reported by Ramsay and Lindner. ${ }^{24}$ They observed strong anisotropic SANS scattering for montmorillonite but not for Laponite solutions under shear. We will refer to this earlier work in the present paper and highlight differences between Laponite and montmorillonite.

Our previous work described a rheological and SANS study and the steady-state behavior of one representative solution of synthetic Laponite and poly(ethylene oxide). ${ }^{25,26}$ The polymer chains were found to be in dynamic adsorption/desorption equilibrium with the clay particles to form a "dynamic network". ${ }^{26}$ The elastic behavior of the network was characterized by constant stress, oscillatory shear, and stress relaxation experiments. Measurements under steady flow characterized the transient behavior of the network. With increasing steady shear rate a pronounced minimum in birefringence was observed, showing that clay platelets orient first, and at a critical shear rate, polymer chains start to stretch. The shear rate dependent viscosity showed near power law behavior and no corresponding critical feature. While bir efringence detects orientational effects on a microscopic length scale, rheology averages over macroscopic changes in the sample. In-situ shear-SANS measurements corroborate the shear-induced orientation of the polymer chains and platelets. ${ }^{25,26}$

The objective of this contribution is to use SANS to investigate the synergistic behavior of viscoelastic polymer-clay solutions for several different concentrations and to provide additional evidence to support the model elucidated from a previous study. ${ }^{25}$

\section{Experimental Section 50}

In the following, we study solutions of the synthetic hectorite type clay, Laponite LRD (Laporte Industries Ltd.), and poly(ethylene oxide) (PEO) $\left(\mathrm{M}_{\mathrm{w}}=10^{6} \mathrm{~g} / \mathrm{mol}\right.$, molecular weight distribution ca. 1.5). The results reported are for highly viscoelastic solutions containing mass fractions of $3 \% L R D$ and 1-5\% PEO at ambient temperature (Table 1 ). The clay a) radial beam

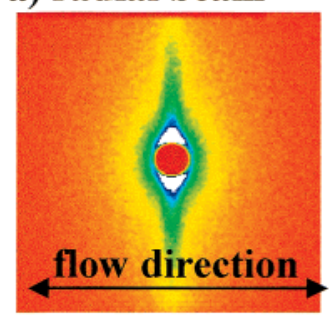

b) tangential beam

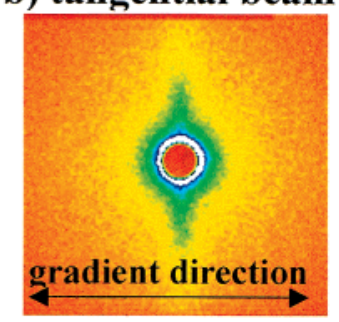

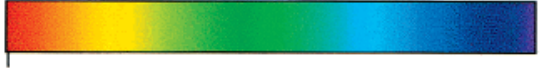

Figure 1. SANS patterns from LRD2 in the (a) radial geometry and (b) tangential geometry at a shear rate of 90 $\mathrm{s}^{-1}$. The apparent anisotropy is lower in tangential beam due to the distribution of platelets caused by the curvature of the shear cell in the tangential scattering geometry. (a) and (b) do not have the same scaling.

produces transparent dispersions of disk-shaped particles ca. $300 \AA$ in diameter and ca. $10 \AA$ thick. ${ }^{39,42} \mathrm{~A} \mathrm{pH}$ of 10 and ionic strength of the solutions were controlled by the addition of $\mathrm{NaOH}$ and $10^{-3} \mathrm{~mol} / \mathrm{L} \mathrm{NaCl}$, respectively. ${ }^{39,42}$

SANS utilized the $30 \mathrm{~m}$ SANS instrument on NG7 Center for Neutron Research at the National Institute of Standards and Technology. ${ }^{43}$ The shear cell as used for solutions is a Couette geometry, which has been described previously. ${ }^{44,45}$ The cell has an i.d. of $60 \mathrm{~mm}$ and a gap of $1 \mathrm{~mm}$, giving a total path length of $2 \mathrm{~mm}$ through the sample. In the standard configuration, referred to as the "radial beam" geometry, the incident beam is parallel to the shear gradient. In a second configuration, referred to as the "tangential beam" geometry, the incident beam is parallel to the flow direction. Sample-todetector distances of 11.25 and $1.75 \mathrm{~m}$ and an incident wavel ength, $\lambda$, of $9 \AA$ were used to give $q$ ranges $(q=4 \pi / \lambda$ $\sin (\theta) / 2)$ of $0.0027 \AA^{-1}<\mathrm{q}<0.0281 \AA^{-1}$ and $0.0137 \AA^{-1}<\mathrm{q}<$ $0.1421 \AA^{-1}$. The primary contrast in the SANS experiment is between $\mathrm{D}_{2} \mathrm{O}$ and the other solution components (clay, PEO, salts); thus, SANS experiments can detect the overall orientation of the solution components under shear. We will refer to these samples as "samples in $\mathrm{D}_{2} \mathrm{O}$ ". Samples in which the solvent is contrast matched to the clay are used to detect the orientation of the polymer chains. SANS contrast variation experiments on LRD2 and LRD4 were performed on 3\% Laponite solutions in a range of $\mathrm{D}_{2} \mathrm{O} / \mathrm{H}_{2} \mathrm{O}$ mixtures at room temperature. The zero scattering point was found to be at 69.8 wt $\% \mathrm{D}_{2} \mathrm{O}$.

\section{Results}

"Samples in $\mathbf{D}_{\mathbf{2}} \mathbf{O}$ ". SANS results (raw data) obtained from LRD2 in the "radial" and "tangential" beam configurations are summarized in Figure 1. Both beam configurations show an anisotropic scattering pattern parallel to the vorticity axis. The apparent scattering is lower in the tangential beam due to the distribution of platelets caused by the curvature of the shear cell in the tangential scattering geometry. The same behavior is observed for several concentrations (Table 1); however, LRD2 is the sample with most distinct features.

Figure 2 summarizes data as obtained from LRD2, LRD3, and LRD4 samples in the radial beam configuration. SANS intensity vs $q$ and vs shear rate is averaged from $10^{\circ}$ sectors parallel to flow and perpendicular to flow (parallel to the vorticity axis). For the same LRD but different PEO concentrations (Table 1) a maximum at roughly $\mathrm{q}_{\max } \approx 0.008 \AA^{-1}$ corresponds to $\mathrm{d}=2 \pi / \mathrm{q}_{\max } \approx 800 \AA$ (Figure 2 ) which is observed at rest. With increasing shear rate the maximum observed 

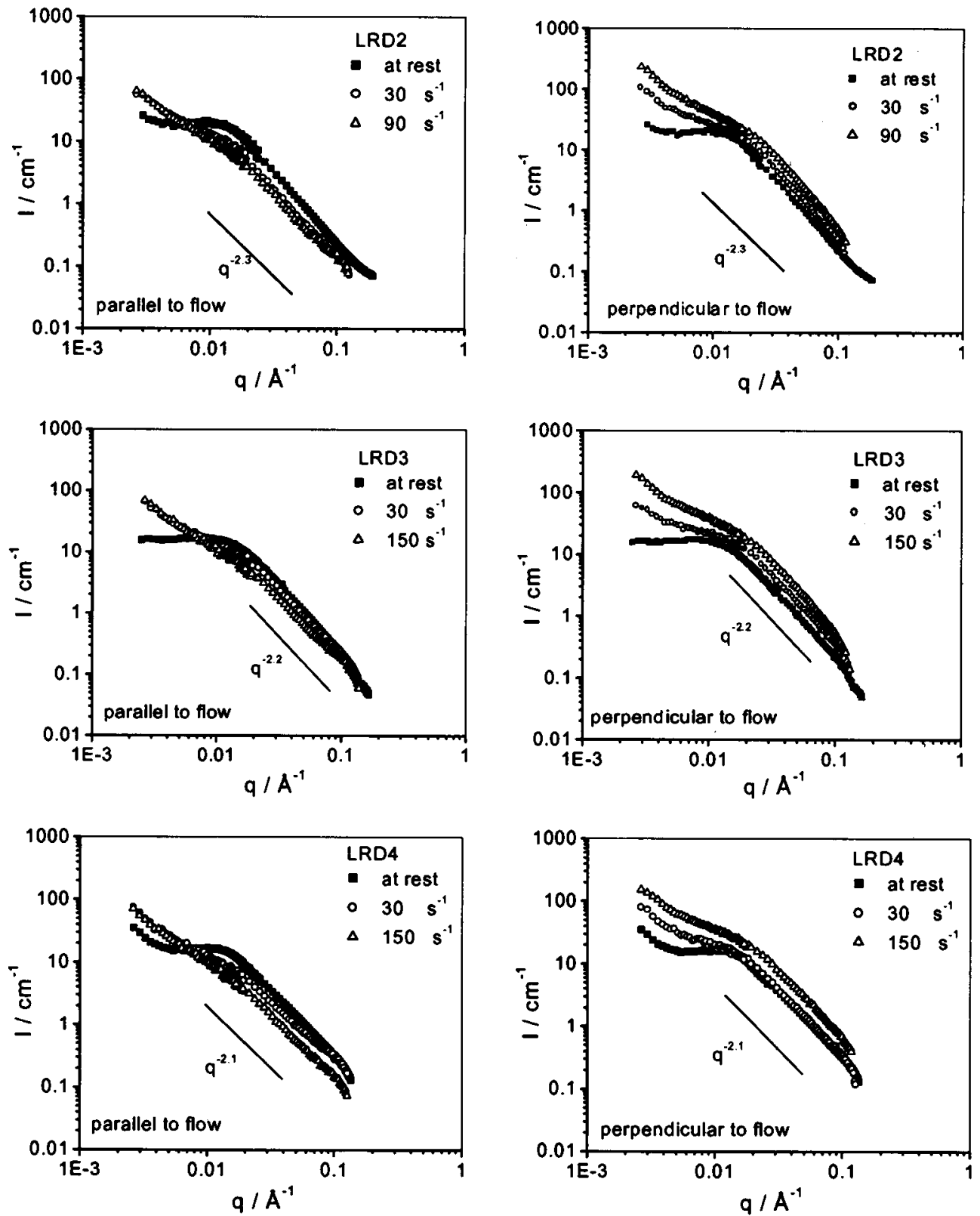

Figure 2. LRD2, LRD3, and LRD4 samples in $\mathrm{D}_{2} \mathrm{O}$ : SANS intensities under static conditions (full squares) in the radial configuration and averaged in $10^{\circ}$ sectors parallel and perpendicular to the flow direction.

with the sample at rest broadens, and we observe the onset of anisotropy in the scattering pattern with a systematic increase of the intensity in the vorticity direction. Intensity increases perpendicular to flow in a q range $\approx 0.005 \AA^{-1}$ and decreases parallel to flow in a q range around $0.015 \AA^{-1}<\mathrm{q}<0.1 \AA^{-1}$. In the high $\mathrm{q}$ range we note that $\mathrm{I}(\mathrm{q})$ scales with $\mathrm{q}^{-2}$. With the samples at rest and increasing PEO concentration the linear region I (q) scales with $q^{-2.3}$ for LRD2, $q^{-2.2}$ for LRD3, $\mathrm{q}^{-2.1}$ for LRD4, and $\mathrm{q}^{-1.8}$ for LRD5 (Figures 2 and 3 ). Figure 3 shows I vs q from two representative samples in a larger q range. The lack of higher order peaks is observed in SANS as well as SAXS up to $q=$ $0.7 \AA^{-1}$

Depending on $\mathrm{pH}$ and salt concentration, LRD1 samples (not shown here) become turbid at low shear rates and turn back to clear upon cessation of shear. At shear rates $>10 \mathrm{~s}^{-1}$ we observe slip at the wallsample interfaces. At these shear rates $\left(>10 \mathrm{~s}^{-1}\right)$ an aqueous thin film is built between the shear cell walls

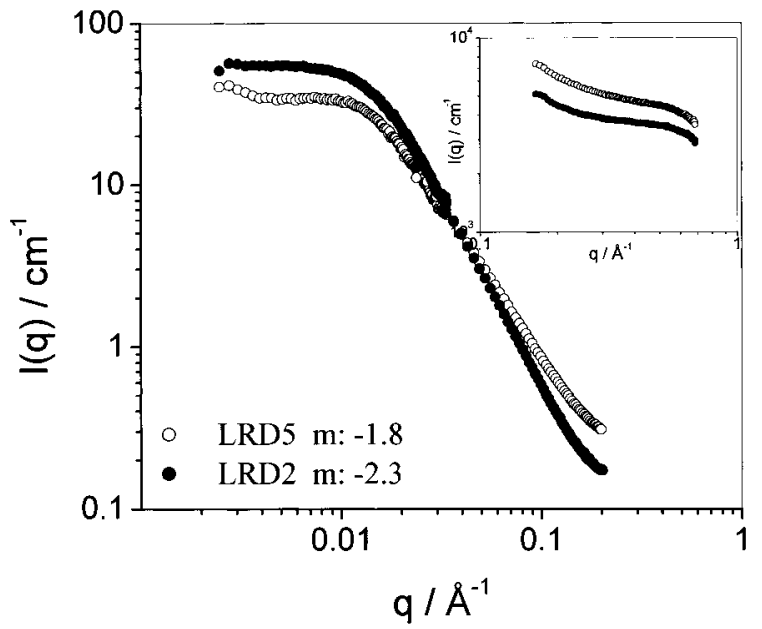

Figure 3. Circularly averaged I (q) vs q plots for LRD2 and LRD5 samples in $\mathrm{D}_{2} \mathrm{O}$ under quiescent conditions. 

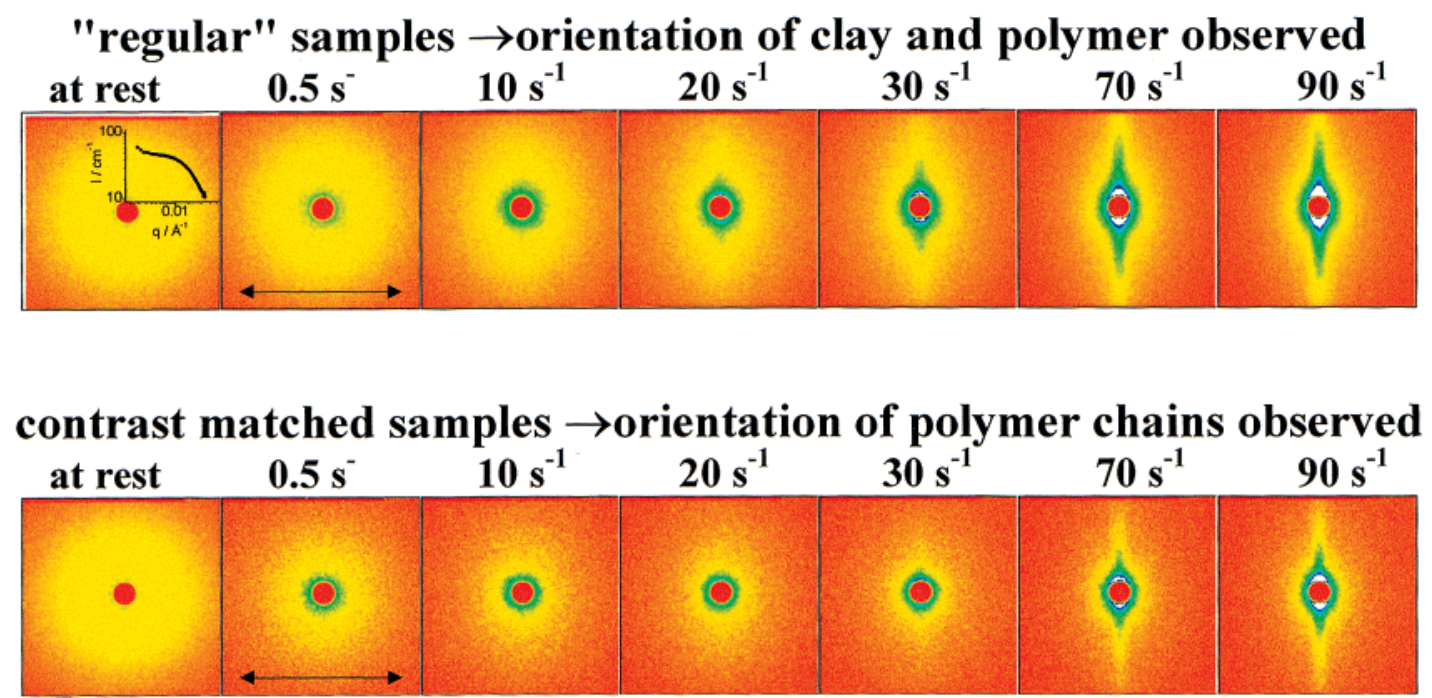

Figure 4. Radial beam configuration: two-dimensional SANS profiles obtained from LRD2 samples in $\mathrm{D}_{2} \mathrm{O}$ (first series, same scaling) and LRD2 contrast matched samples (second series, same scaling).

and the sample due to shear-induced phase separation. SANS anisotropy was observed at shear rates of $150 \mathrm{~s}^{-1}$; however, no quantitative data could be obtained due to possible wall slip effects. This effect could be detected when impurity particles in the sample did not move compared to a mark on the quartz cell.

"Contrast Matched Samples". SANS as obtained from samples in $\mathrm{D}_{2} \mathrm{O}$ and contrast matched samples are shown in Figure 4. Both LRD2 samples show similar scattering patterns under shear (Figures 4) except for the difference in shear rates. Intensity is increased perpendicular to flow and decreases parallel to flow. Comparing both sets of data in Figure 4 leads us to the observation that anisotropy starts at around $5 \mathrm{~s}^{-1}$, with samples in $\mathrm{D}_{2} \mathrm{O}$ and around $30 \mathrm{~s}^{-1}$ with contrast matched samples.

A Guinier analysis of the quiescent LRD2 contrast matched sample yields an apparent " $\mathrm{R}_{\mathrm{g}}$ " of $\mathrm{PEO}$ chains on the order of $400 \AA$ (Figure $5 a$ ). With increasing shear rate a linear regi on devel oped, and the scattering function was characterized by an asymptotic behavior I (q) $\propto q^{-5 / 3}$ at low $q$ and $I(q) \propto q^{-1}$ at high $q$ (Figure $\left.5 b, c\right)$. LRD4 however did not show any linear regime, but a shoulder at a $\mathrm{q}_{\max } \approx 0.01 \AA^{-1}$ and anisotropy developed only at high shear rates and in a certain q range.

"Reference Samples". Reference samples such as pure LRD or PEO solutions (Table 1) did not develop any kind of anisotropy with shear rate (Figure $6 a, b)$. A LRD solution containing 3\% of clay showed a slight change in slope with increasing shear rate and no distinct maximum as observed at higher concentration. A 3\% solution of PEO showed no maximum either and was characterized by $\mathrm{I}(\mathrm{q}) \propto \mathrm{q}^{-2}$ in the low $\mathrm{q}$ range.

\section{Discussion}

Our current understanding of the previous rheology 26 and the present SANS results is that the polymer chains are in a dynamic adsorption/desorption equilibrium with the clay particles to form a network 25,26 (Figure 7). A $3 \%$ reference clay solution is highly viscous, and the vial can be inverted without the solution flowing under the influence of gravity. A 3\% reference polymer solution (PEO: $10^{6} \mathrm{~g} / \mathrm{mol}$ ) is above the overlap concentration. All polymer-clay solutions (Table 1 ) consist of a network between randomly oriented clay platelets and PEO chains with the polymer chains as dynamic cross-links between the platelets. ${ }^{26}$ Results obtained from the polymer-clay solutions in the "radial" and "tangential" beam configurations indicate that flow is sufficient to induce orientation of the polymer-clay network. SANS patterns from both configurations (Figure 1) indicate the shear flow results in an unusual alignment of clay platelets: the platelets are oriented in the flow direction with the surface normal in the vorticity direction (Figure 8). ${ }^{25}$

SANS results obtained from LRD2, LRD3, and LRD4 samples in $\mathrm{D}_{2} \mathrm{O}$ are shown in Figure 2. With the sample at rest a maximum in scattering intensity was observed which broadens at low shear rates. The maximum at $\mathrm{q}_{\max } \approx 0.008 \AA^{-1}$ corresponds to an average spacing between platel ets $d=2 \pi / q_{\max } \approx 800 \AA$ (Figure 7a). On the basis of previous SANS measurements in the absence of polymer, such maxima have been observed before and have been attributed to the devel opment of strong interactions between particles which have parallel orientation with respect to each other ${ }^{24}$ Our polymerclay solutions contain particles which are screened from each other by polymer. Recent work of Saunders et al. ${ }^{35}$ on unoriented pure Laponite gels under similar conditions showed a structure factor maximum at $0.014 \AA^{-1}$ (4\% clay) and $0.02 \AA^{-1}$ ( $6 \%$ clay). Compared to our results, the interparticle distance observed in this study is consistent with their results. With all our samples having the same Laponite (LRD) concentration it is obvious that in a q region sensitive to the dimension of clay platelets $q_{\max }$ does not change much. The peak position in the quiescent scattering patterns (F igure 2) is an indication of an average spacing between platelets $(\approx 800 \AA)$. At low shear rates the peak broadens for LRD2, LRD3, and LRD4 due to nonuniform interparticle spacing (Figure $7 a, b)$ and the low-q intensity increases. At this point polymer chains are more or less isotropic. The model described in Figure 7 suggests that the polymer chains are tethered to the clay particles but does not imply that the chain ends preferentially adsorb to the clay surface. In the high q range we note that I (q) scales with $\mathrm{q}^{-2}$, characteristic of the scattering from randomly oriented disks. A power law decay of $\mathrm{q}^{-2}$ was also observed by Ramsay and Lindner for pure Laponite and montmorillonite solutions which comes from the 

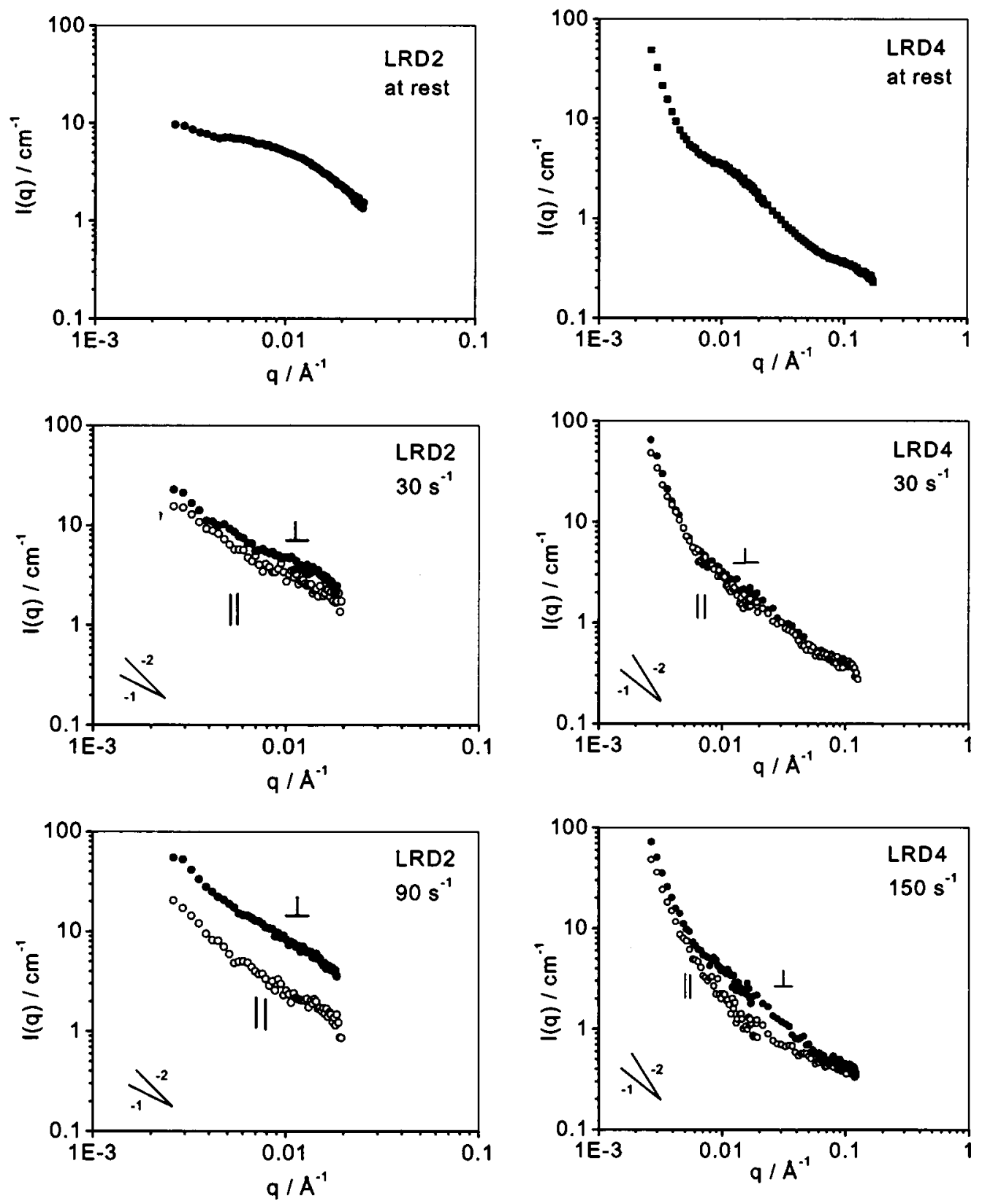

Figure 5. LRD2 (left) and LRD4 (right) contrast matched samples.

form factor for thin disks. ${ }^{24} \mathrm{~A}$ deviation from $\mathrm{q}^{-2}$ is observed in the low q range (Figure 2 ). This deviation is due to the orientation of clay platelets under shear. At low shear rates clay platelets are agile and may tumble or rotate inside the polymer-clay network, which leads to a broadening of the intensity along the azimuthal trace (Figure 4). At higher shear rates polymer chains start to stretch and restrict the mobility of the platelets (Figure 7d). Excess PEO as in LRD4 also restricts the mobility of the platelets, leading to easier orientation (Figure 2). On cessation of shear, the stress on the network decays almost immediately, and the recovery of the structure is control led by the relaxation of the elastic properties of the stretched chains. As the chains retract, the coupling of the polymer to the clay allows the platelets to randomize in orientation under the local viscous environment. The recovery from the SANS anisotropy and from flow bi refringence ${ }^{26}$ is much faster than expected from simple Brownian motion of only the clay particles in a medium of the same viscosity as the polymer-clay solution exhibited macroscopically and is indicative of the dynamic coupling of the polymer chains to the clay. Attempting similar experiments with a different molecular weight PEO $\left(10^{4} \mathrm{~g} / \mathrm{mol}\right.$, molecular weight distribution $<1.5$ ) does not show anisotropy in SANS or strong flow birefringence. We presume that at given concentrations low molecular weight PEO is not sufficient to build up a strong network.

With constant LRD and increasing PEO concentration the linear regi on of I (q) scales with $\mathrm{q}^{-2.3}$ for LRD2, $\mathrm{q}^{-2.2}$ for LRD3, $q^{-2.1}$ for LRD4, and $q^{-1.8}$ for LRD5 with the sample at rest (Figures 2 and 3 ). The scaling factor $x$ in $\mathrm{q}^{\mathrm{X}}$ is PEO dependent. A small-angle neutron scattering study by Lal and Auvray described the adsorption of polymer chains to clay platelets at low polymer and clay concentrations. ${ }^{46,47} \mathrm{~F}$ or low concentrations they have been able to separate the SANS contributions from bulk and adsorbed polymer chains using contrast variation. ${ }^{46,47}$ In our case neither regular samples nor contrast matched samples can distinguish between the 

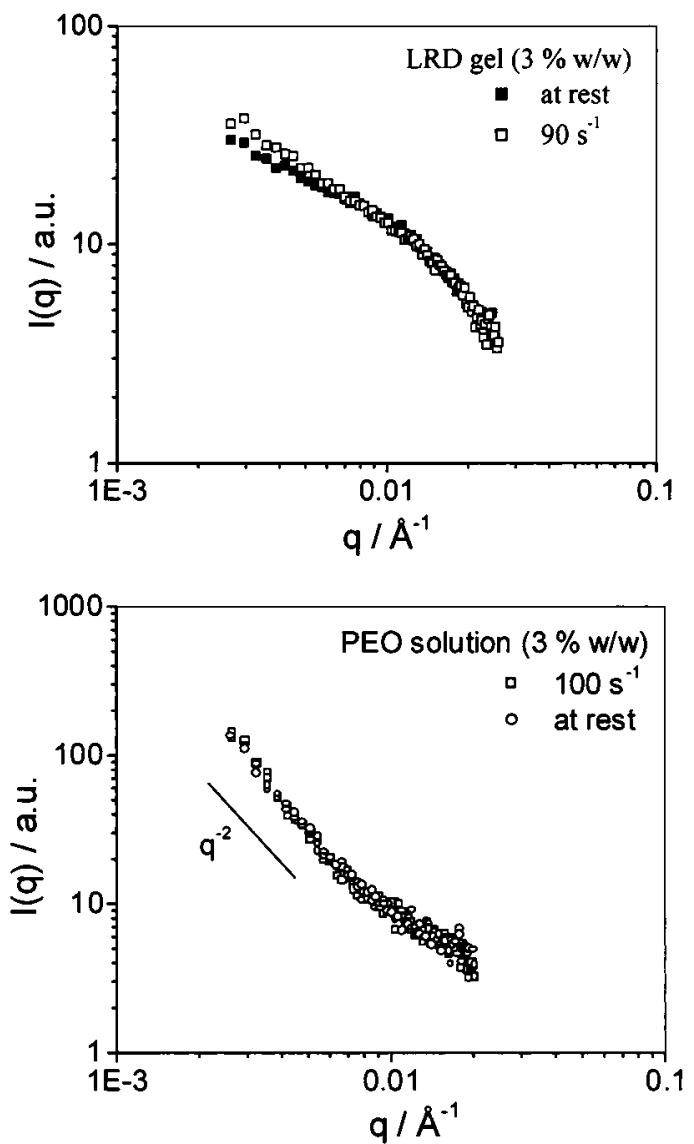

Figure 6. Circularly averaged I (q) vs $q$ for (a) the pure Laponite solution (3\%) and (b) the pure PEO solution (3\%) at rest and under shear.

SANS intensity contribution of network active PEO, adsorbed PEO, or excess PEO at used concentrations (Table 1).

PEO concentration dependence is showing that the polymer is able to keep clay platelets apart at a specified correlation length and at the same interparticle spacing. Figure 3 shows the influence of PEO on LRD2 and LRD5 in a large q range. The insert in Figure 3 is to demonstrate the SANS intensities up to $q=0.7 \AA^{-1}$ before incoherent scattering correction. The information we obtain from these data is that the clay is completely exfoliated because no peaks are detected in the high q region. SANS in the linear q region seems to be more sensitive to the PEO concentration. Additional SAXS experiments on LRD2 and LRD5 show no significant difference in slopes and therefore no PEO dependence of scattering intensity. SAXS is a scattering method, which is more sensitive to the clay concentration.

Contrast matched LRD2 samples show interesting behavior at rest and under shear (Figures 4, 5). Comparing the data from contrast matched samples, where only the PEO is visible, with the "samples in $\mathrm{D}_{2} \mathrm{O}$ ", where both clay and polymer are visible, leads us to the observation that the clay begins to align first, at around $5 \mathrm{~s}^{-1}$. The PEO does not start aligning until critical shear rates above $30 \mathrm{~s}^{-1}$ (F igures 4, 7c). Since the clay platelets are connected to PEO chains, the lack of internal flexibility of the rigid clay particles and the connection to other plates makes them much easier to align than the flexible polymer chains. At high shear rates both, platelets are oriented and polymer chains are stretched (Figure 7d).
A Guinier analysis of the quiescent contrast matched LRD2 sample (Figure 5a) yields an apparent " $\mathrm{R}_{\mathrm{g}}$ " on the order of $400 \AA$. We presume that the polymer chains are entangeled through the volume and that we will not detect the " $\mathrm{R}_{\mathrm{g}}$ " of one single polymer chain. One possibility is that the apparent " $\mathrm{R}_{\mathrm{g}}$ " observed here represents the dimension of PEO clustered around a platelet particle that builds up the network (platel et diameter is ca. $300 \AA$ ). In this case PEO is not distributed homogeneously in the sample, but we observe density fluctuations. An alternate interpretation is that the scattering is due to "holes" in the polymer/solvent matrix where the "holes" are PEO poor (possibly clay rich) regions. In this case the PEO would be homogeneously entangled through the whole volume.

With increasing shear rate a linear region develops (Figure $5 b, c$ ) with LRD2, and the scattering function is characterized by an asymptotic behavior $\mathrm{I}(\mathrm{q}) \propto \mathrm{q}^{-5 / 3}$ at low $\mathrm{q}$ and $\mathrm{I}(\mathrm{q}) \propto \mathrm{q}^{-1}$ at high $\mathrm{q}$. The $-5 / 3$ exponent corresponds to a perturbed coil with excluded volume, in our case a perturbed soft PEO shell. The -1 exponent at high $q$ is characteristic of scattering from a rigid rod, and its presence in this q range indicates a relatively large effective persistence length (fairly "stiff" chains).

LRD4 contrast matched samples show quite different behavior due to the higher PEO concentration. At rest we observe a shoulder which may be correlated with "holes" in the polymer/solvent matrix where the "holes" are PEO poor (clay rich) regions. The polymer network contains PEO chains connected to the clay and excess PEO chains, which act as a shear thickener. SANS on contrast matched samples cannot distinguish between these two PEO components but gives an "average" intensity. Therefore, SANS intensity perpendicular and parallel to the flow direction is not that different and only weak anisotropy is observed.

Scattering from a $3 \%$ reference sample of PEO, at the same $\mathrm{pH}$ and salt concentration as LRD3, is given in Figure 6. Comparison with Figure 5 a shows the dramatic conformational change in PEO caused by the presence of clay. The PEO reference sample exhibited no anisotropic scattering at shear rates up to $100 \mathrm{~s}^{-1}$ (F igure 6), indicating that the applied shear rate is not high enough to overcome randomizing effects caused by the first normal mode of single chain relaxation (characteristic time $\tau_{1}$ ) when the clay is absent. Preliminary dynamic light scattering (DLS) experiments on a dilute solution of PEO $\left(0.1 \%, M_{w}=10^{6} \mathrm{~g} / \mathrm{mol}\right)$ in pure $D_{2} \mathrm{O}$ result in " $\mathrm{R}_{\mathrm{g}}$ " around $1240 \AA$. DLS on a dilute solution of $\mathrm{PEO}$ in $\mathrm{D}_{2} \mathrm{O}$ at the same $\mathrm{pH}$ and salt concentration as LRD2 show " $\mathrm{R}_{\mathrm{g}}$ " around $1034 \AA .48$ Literature values for the unperturbed " $\mathrm{R}_{\mathrm{g}}$ " of linear PEO chains at $\mathrm{M}_{\mathrm{w}}=$ $10^{6} \mathrm{~g} / \mathrm{mol}$ in water are around $888 \AA .49$

The orientational alignment of the platelets is a competition between (i) flow alignment and (ii) orientational and configurational relaxation. Flow alignment is induced by orientation of platelets and stretching of polymer chains under shear. Orientational and configurational relaxation are caused by a randomization through adsorption/desorption processes at clay surfaces. Increasing adsorption times of the polymer attached to the clay drive the equilibrium toward (i) while desorption favors (ii).

Using arguments similar to Ramsay ${ }^{24}$ as well as Hayter and Penfold, 15 these competing effects can be characterized by $\Gamma_{\mathrm{x}}=\mathrm{g} \tau_{\mathrm{x}}$ with $\mathrm{g}$ being the shear rate and $\tau$ the relaxation time ( $\tau_{0}$ for the adsorption/desorp- 
a)

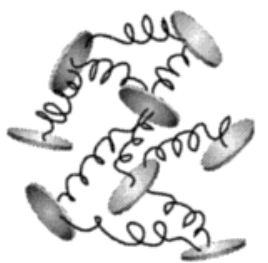

b)

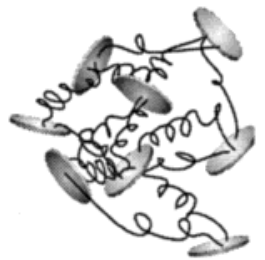

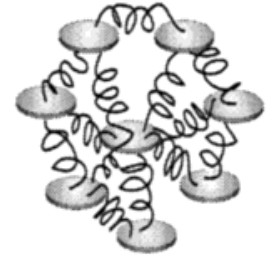

c)

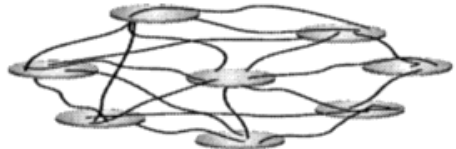

d)

Figure 7. (a) At rest: same interparticle distance. (b) At low shear rates: nonuniform interparticle distance. (c) At critical shear rate: platelets oriented, polymer chains more or less relaxed. (d) At high shear rates: platelets oriented and chains stretched. The model suggests that the polymer chains are tethered to the clay particles but does not imply that the chain ends preferentially adsorb to the clay surface.

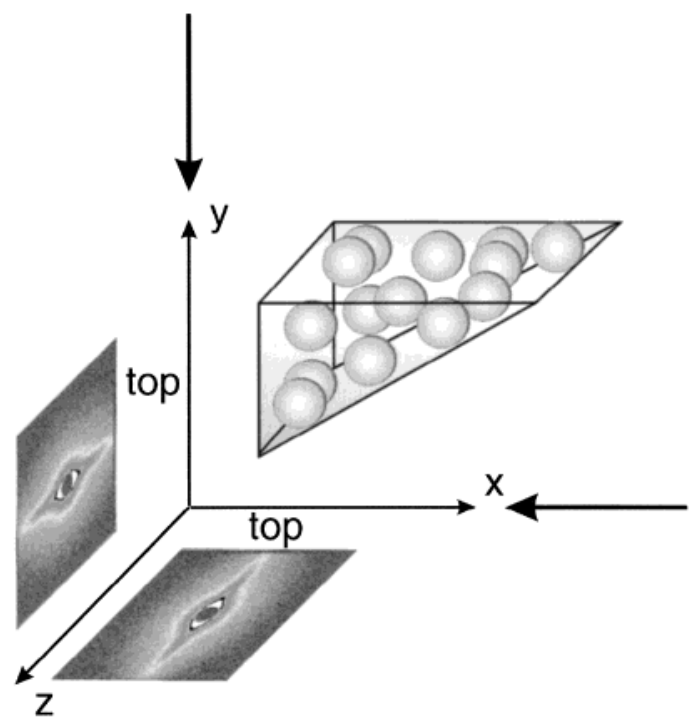

Figure 8. As the platelets align with surface normal along neutral direction, we observe a vertical streak in the radial SANS pattern $(\mathrm{y})$ and a vertical streak in the tangential SANS pattern (x).

tion process, i.e., the attachment time). Full alignment will occur when $\Gamma \gg 1$. SANS on regular samples as well as birefringence show the clay alignment starts at shear rates of $5 \mathrm{~s}^{-1}$. This implies that $\tau_{0} \approx 0.05 \mathrm{~s}$ for $\mathrm{PEO}$ on the Laponite surface under experimental conditions. SANS data on contrast matched samples on the other hand show no evidence of the polymer chains stretching until shear rates of ca. $30 \mathrm{~s}^{-1}$. This is due to the flexibility of the PEO chains, giving them many internal modes of relaxation, which can partially compensate for being tethered to clay particles. Nonetheless, the chains do align at shear rates well bel ow those for chains who are not tethered (reference sample). Future work will compare relaxation times obtained from rheology and flow birefringence with SANS and dynamic light scattering and will correlate the corresponding relaxation times with the normal modes in the polymer-clay system.

Finally, a 3\% aqueous Laponite reference sol ution was measured and showed no evidence of SANS anisotropy (Figure 6). With increasing shear rate a slight change in slope is observed which is due to the breaking up of aggregates and some orientation of the clay. This weak orientation can be detected by flow birefringence ${ }^{26}$ and light scattering ${ }^{39}$ and was attributed to alignment of micron-sized domains rather than individual clay particles. No distinct SANS maximum is detected, as one would observe for higher clay concentrations. ${ }^{35}$ Although flow birefringence experiments on Laponite solutions show some flow birefringence under shear, the corre- sponding degree of orientation is too low as to be detected by anisotropy in SANS. Our results on reference Laponite samples are in agreement with previous SANS studies by Ramsay and Lindner. ${ }^{24}$ They observed strong anisotropic SANS scattering for montmorillonite but not for Laponite solutions under shear. Their measurements have shown that the range of orientational correlation in pure clay dispersions is dependent on the day concentration, particle size, and shape. Thus, it is apparent that orientational correlation is more extensive for the larger and more anisotropic montmorillonite clay particles than for Laponite particles. The preferential alignment was found to persist at distances $\gg 10^{3} \AA .24$

We conclude that the anisotropic SANS pattern observed in the polymer-clay solution is due to the coupling between clay platelets and polymer, allowing a higher orientation than either single component in solution can produce.

\section{Summary}

The influence of shear on viscoelastic polymer-clay solutions was investigated by means of small-angle neutron scattering (SANS) under shear. Polymer chains are in dynamic adsorption/desorption equilibrium with the clay particles to form a network. Under shear we observe an unusual alignment of clay platelets along the flow direction with the surface normal in the vorticity direction. SANS on samples in $\mathrm{D}_{2} \mathrm{O}$ measured the shear-induced orientation of polymer and platelets. SANS on contrast matched samples detected the orientation of the polymer alone. With increasing shear rate clay particles orient first (SANS on samples in $\mathrm{D}_{2} \mathrm{O}$ ), and then polymer chains start to stretch (SANS on contrast matched samples). The recovery from the SANS anisotropy is much faster than expected from simple Brownian motion of only the clay particles in a medium of the same viscosity as the polymer-clay solution exhibited macroscopically and is indicative of the dynamic coupling of the polymer chains to the clay.

Acknowledgment. Financial support by the Alexander von Humboldt Foundation is gratefully acknowledged by Gudrun Schmidt. We thank Dr. R. Cueto for performing preliminary DLS experiments and Prof. P. Russo for using his DLS instrument. We also thank the reviewers for useful suggestions and comments.

\section{References and Notes}

(1) Vaia, R. A.; Gianellis, E. P. MRS Bull. 2001, 62, 394-401.

(2) Manias, E.; Touny, A.; Wu, L.; Stawhecker, K.; Lu, B.; Chung, T. C. Chem. Mater. 2001, 13, 3516-3523.

(3) Lan, T.; Pinnavaia, T. Chem. Mater. 1994, 6, 2216-2219. 
(4) Wang, Z.; Pinnavaia, T. J . Chem. Mater. 1998, 10, 1820-1826.

(5) Okada, A.; Usuki, A. Mater. Sci. Eng. 1995, C3, 109.

(6) Usuki, A.; Kojima, Y.; Kawasumi, M.; Okada, A.; Fukushima, Y.; Kurauchi, T.; Kamigaito, O.J . Mater. Res. 1993, 8, 11791184.

(7) Brown, A. B. D.; Ferrero, C.; Narayanan, T.; Rennie, A. R. Eur. Phys. J . B 1999, 11, 481-489.

(8) Levitz, P.; Lecolier, E.; Mourchid, A.; Delville, A.; Lyonnard, S. Europhys. Lett. 2000, 49, 672-677.

(9) Melton, I. E.; Rand, B. J . Colloid InterfaceSci. 1977, 60, 321330.

(10) Ramsay, J. D. F. J . Colloid Interface Sci. 1986, 109, 441447.

(11) Sondergaard, K.; Lyngaae-J orgensen, J. Rheo-Physics of Multiphase Polymer Systems; Technomic: Lancaster, PA, 1995.

(12) Nakatani, A. I. In Flow-Induced Structure in Polymers; Nakatani, A. I., Ed.; American Chemical Society: Washington, DC, 1995; Vol. 579, pp 1-19.

(13) Larson, R. G. TheStructureand Rheol ogy of Complex Fluids; Oxford University Press: New York, 1999.

(14) Fuller, G. G. Optical Rheometry of Complex Fluids; Oxford University Press: Oxford, 1995.

(15) Hayter, J . B.; Penfold, J . J . Phys. Chem. 1984, 88, 45894593.

(16) Chen, Z. R.; Kornfield, J . A. Polymer 1998, 39, 4679-4699.

(17) Koppi, K. A.; Tepe, T.; Schulz, M. F.; Zhao, J .; Tirrell, M.; Bates, F. S.; Mortensen, K.; Almdal, K. Abstr. Pap.-Am. Chem. Soc. 1995, 209, 145-Poly.

(18) Koppi, K. A.; Tirrell, M.; Bates, F. S.; Almdal, K.; Mortensen, K. J. Rheol. 1994, 38, 999-1027.

(19) Tirrell, M.; Koppi, K.; Tepe, T.; Bates, F. S. Abstr. Pap.-Am. Chem. Soc. 1994, 207, 356-Poly.

(20) Brown, A. B. D.; Clarke, S. M.; Convert, P.; Rennie, A. R. J . Rheol. 2000, 44, 221-233.

(21) Mourchid, A.; Delville, A.; Lambard, J .; Lecolier, E.; Levitz, P. Langmuir 1995, 11, 1942-1950.

(22) Pignon, F.; Magnin, A.; Piau, J . M. Phys. Rev. Lett. 1997, 79, 4689-4692.

(23) Pignon, F.; Magnin, A.; Piau, J . M. J . Rheol. 1998, 42, 13491373.

(24) Ramsay, J . D. F.; Lindner, P. J . Chem. Soc., Faraday Trans. 1993, 89, 4207-4214.

(25) Schmidt, G.; Nakatani, A. I.; Butler, P. D.; Karim, A.; Han, C. C. Macromol ecules 2000, 33, 7219-7222.

(26) Schmidt, G.; Nakatani, A. I.; Han, C. C. Rheol. Acta 2002 $41,45-54$

(27) van Olphen, H. Discuss. Faraday Soc. 1951, 82.

(28) van Olphen, H. An Introduction to Clay Colloid Chemistry, 2nd ed.; J ohn Wiley and Sons: New Y ork, 1977.

(29) Dijkstra, M.; Hansen, J. P.; Madden, P. A. Phys. Rev. E 1997, 55, 3044-3053.
(30) Dijkstra, M.; Hansen, J . P.; Madden, P. A. Phys. Rev. Lett. 1995, 75, 2236-2239.

(31) Mourchid, A.; Delville, A.; Levitz, P. Faraday Discuss. 1995, 275-285

(32) Norrish, K. Discuss. Faraday Soc. 1954, 120-134.

(33) Rand, B.; Melton, I. E. J . Colloid InterfaceSci. 1977, 60, 308320.

(34) Rand, B.; Peknec, E.; Goodwin, J . W.; Smith, R. W. J . Chem. Soc., Faraday Trans. 1 1980, 76, 225-235.

(35) Saunders, J. M.; Goodwin, J. W.; Richardson, R. M.; Vincent, B. J . Phys. Chem. B 1999, 103, 9211-9218.

(36) Gabriel, J. . C. P.; Sanchez, C.; Davidson, P. J . Phys. Chem. 1996, 100, 11139-11143.

(37) Mourchid, A.; Lecolier, E.; Van Damme, H.; Levitz, P. Langmuir 1998, 14, 4718-4723.

(38) Pignon, F.; Piau, J . M.; Magnin, A. Phys. Rev. Lett. 1996, 76, 4857-4860.

(39) Pignon, F.; Magnin, A.; Piau, J . M.; Cabane, B.; Lindner, P.; Diat, O. Phys. Rev. E 1997, 56, 3281-3289.

(40) Bonn, D.; Kellay, H.; Tanaka, H.; Wegdam, G.; Meunier, J. Langmuir 1999, 15, 7534-7536.

(41) Bihannic, I.; Michot, L. J .; Lartiges, B. S.; Vantelon, D.; Labille, J .; Thomas, F.; Susini, J .; Salome, M.; Fayard, B. Langmuir 2001, 17, 4144-4147.

(42) Ramsay, J. D. F.; Swanton, S. W.; Bunce, J . J . Chem. Soc., Faraday Trans. 1990, 86, 3919-3926.

(43) Glinka, C. J ; Barker, J. G.; Hammouda, B.; Krueger, S.; Moyer, J . . .; Orts, W. J . J . Appl. Crystallogr. 1998, 31, 430445.

(44) Straty, G. C.; Hanley, H. J . M.; Glinka, C. J . J . Stat. Phys. 1991, 62, 1015-1023.

(45) Straty, G. C.; Muzny, C. D.; Butler, B. D.; Lin, M. Y.; Slawecki, T. M.; Glinka, C. J .; Hanley, H. J . M. Nud. Instrum Methods A 1998, 408, 511-517.

(46) Lal, J .; Auvray, L. J . Appl. Crystallogr. 2000, 33, 673-676.

(47) Lal, J .; Auvray, L. Mol. Cryst. Liq. Cryst. 2001, 356, 503515.

(48) Preliminary DLS experiments were performed by Dr. R. Cueto at Louisiana State University in the laboratory of Prof. P. Russo.

(49) Brandup, J .; Immergut, E. H.; Grulke, E. A. Polymer Handbook; J ohn Wiley and Sons: New York, 1999.

(50) Certain equipment and instruments or materials are identified in this paper in order to adequately specify the experimental details. Such identification does not imply recommendation by the National Institute of Standards and Technology nor does it imply the materials are the best available for the purpose.

MA0115141 